\title{
Technical development in cardiac CT: current standards and future improvements - a narrative review
}

\author{
Patrizia Toia ${ }^{1}$, Ludovico La Grutta ${ }^{2}$, Giulia Sollami ${ }^{1}$, Alberto Clemente ${ }^{3}$, Cesare Gagliardo ${ }^{1}$, \\ Massimo Galia ${ }^{1}$, Erica Maffei ${ }^{4}$, Massimo Midiri ${ }^{1}$, Filippo Cademartiri ${ }^{5} \wedge$
}

${ }^{1}$ Department of Biomedicine, Neurosciences and Advanced Diagnostic (BiND), University of Palermo, Palermo, Italy; ${ }^{2}$ Department of Health Promotion Sciences Maternal and Infantile Care, Internal Medicine and Medical Specialities (ProMISE), University of Palermo, Palermo, Italy; ${ }^{3}$ Fondazione Toscana G. Monasterio CNR - Regione Toscana, Pisa and Massa, Italy; ${ }^{4}$ Department of Radiology, Area Vasta 1, ASUR Marche, Urbino (PU), Italy; ${ }^{5}$ Department of Radiology, SDN IRCCS, Naples, Italy

Contributions: (I) Conception and design: All authors; (II) Administrative support: None; (III) Provision of study materials or patients: None; (IV) Collection and assembly of data: All authors; (V) Data analysis and interpretation: All authors; (VI) Manuscript writing: All authors; (VII) Final approval of manuscript: All authors.

Correspondence to: Filippo Cademartiri, MD, PhD, FESC, FSCCT. Department of Radiology, SDN IRCCS, Naples, Italy.

Email: filippocademartiri@gmail.com.

\begin{abstract}
Non-invasive depiction of coronary arteries has been a great challenge for imaging specialists since the introduction of computed tomography (CT). Technological development together with improvements in spatial, temporal, and contrast resolution, progressively allowed implementation of the current clinical role of the CT assessment of coronary arteries. Several technological evolutions including hardware and software solutions of CT scanners have been developed to improve spatial and temporal resolution. The main challenges of cardiac computed tomography (CCT) are currently plaque characterization, functional assessment of stenosis and radiation dose reduction. In this review, we will discuss current standards and future improvements in CCT.
\end{abstract}

Keywords: Coronary artery disease (CAD); cardiac computed tomography (CCT); atherosclerosis; diagnosis; prognosis; therapy

Submitted May 22, 2020. Accepted for publication Sep 18, 2020.

doi: $10.21037 / \mathrm{cdt}-20-527$

View this article at: http://dx.doi.org/10.21037/cdt-20-527

\section{Introduction}

The depiction of coronary arteries has been a great challenge for imaging specialists since the introduction of computed tomography (CT); the heart is a rapidly moving organ and coronary arteries have a small diameter. Several technological innovations have followed since the introduction of the $1^{\text {st }}$ generation of CT in 1972. One of the most important improvements was the increase in rotation speed, which translated into better temporal resolution and spatial resolution along the $\mathrm{z}$ axis; this was made possible by the use of submillimeter collimations with extended volumetric acquisitions. With the introduction of 16-slice CT scanners and soon after of 64-slice CT scanners, the first encouraging results in the cardiac field were achieved (1).

Cardiac computed tomography (CCT) was originally compared with invasive coronary angiography (ICA), although the two techniques present different targets. However, clear advantages of non-invasive CCT technique should be highlighted, despite a better spatial $(0.1-0.2 \mathrm{~mm}, 50 \mathrm{lp} / \mathrm{cm})$ and temporal resolution ( $<20 \mathrm{~ms}$ ) of ICA (2).

Technological progress together with spatial, temporal and contrast resolution improvements, have progressively

\footnotetext{
$\wedge$ ORCID: 0000-0002-0579-3279.
} 
Table 1 Main resolution challenges for CCT

\begin{tabular}{ll}
\hline Issue & Challenges \\
\hline Spatial resolution & $\begin{array}{l}\text { Evaluation of small distal coronary arteries and branches; assessment of calcified plaques and degree of stenosis } \\
\text { (blooming); evaluation of intra-stent lumen; analysis of plaque characteristics (area, volume, attenuation) }\end{array}$ \\
Temporal resolution & Cardiac motion artifacts; high heart rate; arrhythmias \\
Contrast resolution & Intracoronary opacification; coronary plaque imaging \\
\hline
\end{tabular}

$\mathrm{CCT}$, cardiac computed tomography.

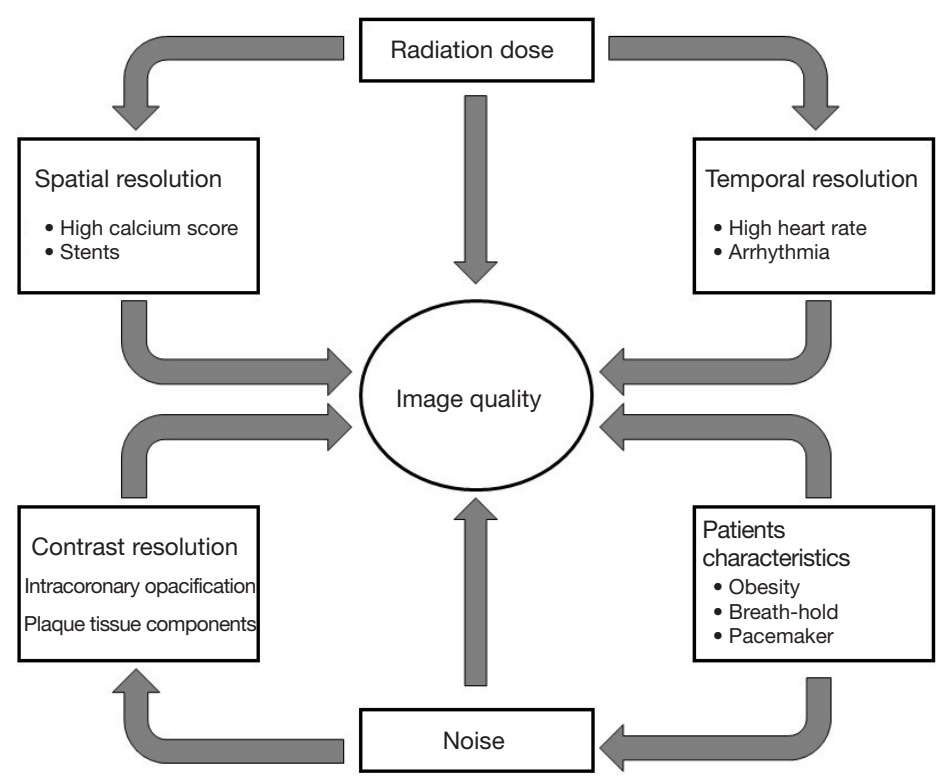

Figure 1 Different factors influencing and converging towards imaging quality in cardiac CT.

implemented image quality and robustness of CCT (Table 1) (Figure 1). Current challenges of CCT now focus on plaque characterization, functional studies and dose reduction.

In this review, we discuss current standards and the future improvements in CCT.

We present the following article in accordance with the Narrative Review reporting checklist (available at http:// dx.doi.org/10.21037/cdt-20-527).

\section{Methods}

Information sources used to write this paper were: Medline search, hand searches of the references of retrieved literature, web search, libraries and personal experience.

\section{Spatial resolution}

Spatial resolution is defined as the ability to distinguish 2 neighbouring structures as separated. It is influenced by several factors: detectors, thickness, pitch, reconstruction parameters, filters and artifacts. Insufficient spatial resolution may affect the capability of evaluation of coronary arteries, especially of more distal (and therefore small) branches as well as coronary stenosis detection and quantification. Furthermore, insufficient spatial resolution may result in partial volume artifacts, which may also cause blooming artifacts and overestimation of coronary artery stenosis, with the generation of false positives and reduced specificity (3). Therefore, spatial resolution was the primary hardware issue to be developed, first to allow and then to improve, the visualization of coronary arteries.

Detectors are among the main determinants of spatial resolution. The detector is an X-ray sensor that converts incoming photons, that have passed through the object of study, in an electrical signal detected from data acquisition system; this signal is collected, amplified and converted into a 
digital information through an analog-to-digital converter (4). The raw data, by a complex system of mathematical calculations, will be then associated with corresponding space position identified by an axes system ( $\mathrm{x}, \mathrm{y}$, and $\mathrm{z}$ ) (4). The $\mathrm{x}$ and $\mathrm{y}$ axes correspond to the transverse plane (i.e., axial), representing the sides of the pixel that form a matrix; the $\mathrm{z}$ axis corresponds to the longitudinal axis and is reflected by slice thickness; the 3 measures of the voxel create three-dimensional object. The elementary unit of volumetric imaging is the voxel, which should be as isotropic (cubic) as possible in cardiac applications (4).

Spatial frequency with the unit of "line pairs per centimeter" is used for object dimension measurement (5).

The increase of the detectors' rows determines an increase of global z-axis (i.e., longitudinal) coverage, which depends on the width of the detector and on the pitch. With increasing detector z-coverage, few rotations are needed to cover the cardiac volume (6).

The first turning point of cardiac imaging with CT was the shift from 4- to 16-slice CT scanner detectors with an improved collimation and $\mathrm{z}$-axis coverage; the minimum spatial resolution increased from 1 to $0.6 \mathrm{~mm}$ (7). The trend, known as "slice's war" (8), was to increase the number of the detector rows in subsequent years (8). From 64-slice CT to 128 -slice scanner the spatial resolution improved from 0.5 to $0.4 \mathrm{~mm}$, until $0.35 \mathrm{~mm}$ in a 320 -slice CT scanner and $0.17 \mathrm{~mm}$ in 640 -slice CT $(7,9)$. Furthermore, some manufacturers, have developed a CT with 128 detectors' slices that deliver the equivalent of a 256-slice $(160 \mathrm{~mm}$ ) by using z-flying focal spot system (8); this system allows a fluctuation of the focal spot in the $\mathrm{z}$-axis, in order to obtain two overlapping slices for each projection (10).

It seems apparently easy the imagine that by simply increasing the number of detector rows one could cover the range of the heart in a single heart-beat without moving the CT table; however, when the detector is very wide there is a progressive deformation of the reconstructed object that is very little at the isocenter of the scanner and that becomes very severe in the peripheral regions of the field of view (FOV). Correction algorithms have been developed through the years, but this issue is not completely solved and in fact most very wide detector scanner cannot use the full detector when applied to conventional body CT applications.

$\mathrm{X}$-ray scatter is another challenge of larger detector z-coverage (6). The same problem occurs with the flat panel CT (FPCT). The FPCT is characterized by a single large detector (consisting of scintillation crystals based on cesium iodide and an amorphous selenium matrix), that allows a large coverage of the $z$-axis, with a matrix of $40 \times 30 \mathrm{~cm}$ divided into $2048 \times 1536$ voxels, but a low contrast resolution caused by the $\mathrm{X}$-ray scatter (8).

From the first 4-slice CT scanner the z-coverage significantly increased; thus, resulting in shorter breathholding requirements for heart scanning. This led to the consequence that the detector coverage was no longer a significant limitation of CCT; however, it must be kept in mind that the number of heartbeats that contributes to the constitution of the image decreases as the detector $\mathrm{z}$-coverage increases. A detector with large $\mathrm{z}$-coverage also has a huge advantage in the analysis of dynamic myocardial perfusion (11).

Detectors have followed CT technological evolution, in order to support the increase in the acquisition speed and the tube peak power. Detectors must have several features to deliver a good diagnostic image quality: accuracy, dynamic range, stability, uniformity, speed of response, fast afterglow, resolution, geometric efficiency, detector quantum efficiency, and cross-talk (12). The increased gantry speed required detector's changes with improved speed, performance and power. Detector cells and focal spots decreased in size to ameliorate spatial resolution; the anode angle increased and the physical power density in the focal spot was intensified (13). The in-plane spatial resolution of a CT system is limited by the number of active detector channels in a detector row and by their involvement (6). The effects of detectors size have become crucial in spatial resolution and have been one of the research elements for the technological advancement of CT in recent decades. A greater volume to cover and the need to have sub-millimetric acquisitions require more detectors in the $\mathrm{z}$ axis (14). For a long time, the size of clinical detectors has remained unchanged with a value of $0.5-0.625 \mathrm{~mm}$ at the isocenter. In a phantom study, Onishi et al. (15) evaluated in-stent restenosis with a clinical CT scanner with energy integrating detectors of $0.25 \mathrm{~mm}$ dimension at isocenter. The focal spot size should be reduced to have a higher spatial resolution with reduced detector size (15). Another option to improve spatial resolution is to expand the detector sampling with the z-flying spot (16-18).

Another basic requirement for coronary artery visualization is the isotropic sub-millimetric spatial resolution (19). The in-plane spatial resolution of a CT scanner is affected by the number of active channels of the detectors and their involvement. Modern CT scanners use techniques such as quarter detector offset or in-plane flying focal spot, in order to double the number of samples 

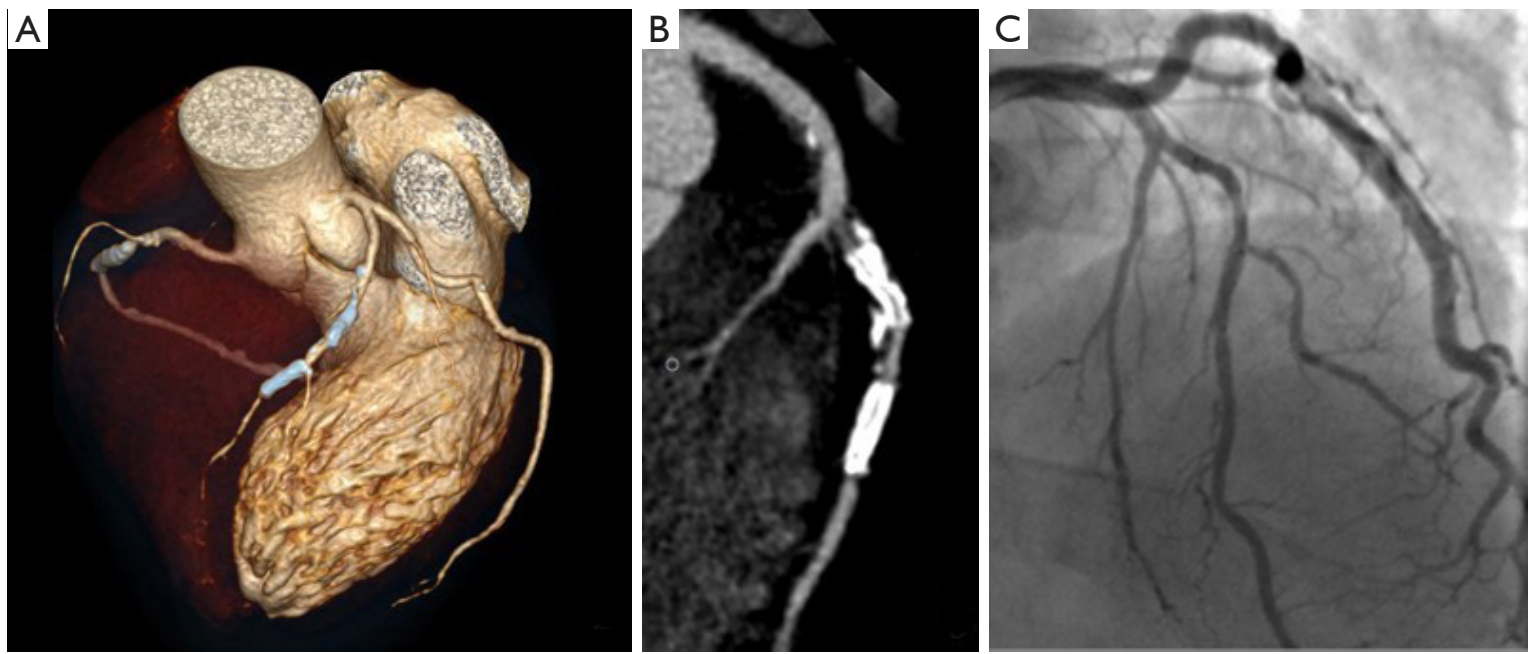

Figure 2 Case of false positive displaying intrastent restenosis at cardiac computed tomography (CCT). (A) Volume rendering; (B) multiplanar reconstruction of the left anterior descending coronary artery; (C) corresponding conventional angiogram depicting intrastent patency.

acquired in the scan FOV and improve spatial resolution. The quarter detector offset is used to reduce aliasing artifacts (19). The in-plane flying focal spot is able to double the number of samplings carried out by moving the position of the focal spot in the anode plate (6).

One of the advantages of 64-slice CT scanners, over the past, was an improved resolution along the z-axis; indeed, even thinner collimated slices have become available. This evolution was crucial for coronary arteries evaluation. However, the spatial resolution of 64-slice scanners was not optimal for clinical evaluation of intra-stent lumen; instead, it allows a correct evaluation of coronary stenoses, by-pass and cardiac anatomy. McCollough et al. (20) demonstrated, in a phantom model, that blocking detectors in fan angle direction together with a deconvolution technique in the longitudinal direction and iterative reconstruction, allowed a good longitudinal resolution with improved dose efficiency.

Nonetheless, in-stent evaluation remains a challenge in CCT, especially in case of hyperdense (e.g., not fully deployed) and small stents (Figure 2). The composition of the detectors has also changed in the years; their composition has improved CT performances over the last years. Initially the detectors were gaseous, mainly xenon detectors (21). However, gaseous detectors were lowperforming, so today exclusively solid-state CT detectors are used. A solid-state CT detector can be made with CdWO4, yttrium and gadolinium $\left(\mathrm{Gd}_{2} \mathrm{O}_{2} \mathrm{~S}\right)$ ceramics (21), and it can be used in the fourth-generation CT thanks to its conformation. Many new materials have been tested to optimize the performance of the detectors; among these a garnet-based substance detector was conceived. This detector shows a noise reduction and a spatial and temporal resolution improvement compared to gadolinium ceramic detector with a 100-times faster decay time (defined as the speed of light emission after the excitation) and $75 \%$ shorter afterglow (defined as the time needed to reset the detector for a new excitation) (22). The garnet-based detector can be used also in the dual-energy CT (DECT), allowing simultaneous acquisition of multiple energy levels from an X-ray tube (22). These novel features can be exploited in cardiac imaging, in which more precise and faster performances are fundamental. It has also been shown that garnet-based detectors provide an improvement in the evaluation of coronary stents due to the reduction of artifacts (22).

Among the areas of research of recent years, we must include the Photon-Counting Detector CT (PCD). PCD represents an emerging technology that allows a reduction of electronic noise, beam-hardening and metal artifacts with increased contrast-to-noise ratio and an extremely high spatial resolution (33 line pairs per centimeter); all these features make it potentially applicable in the cardiovascular field, especially for stent imaging (23).

For a long time, matrix has maintained the standard dimensions of $512 \times 512$ in most of clinical applications, 

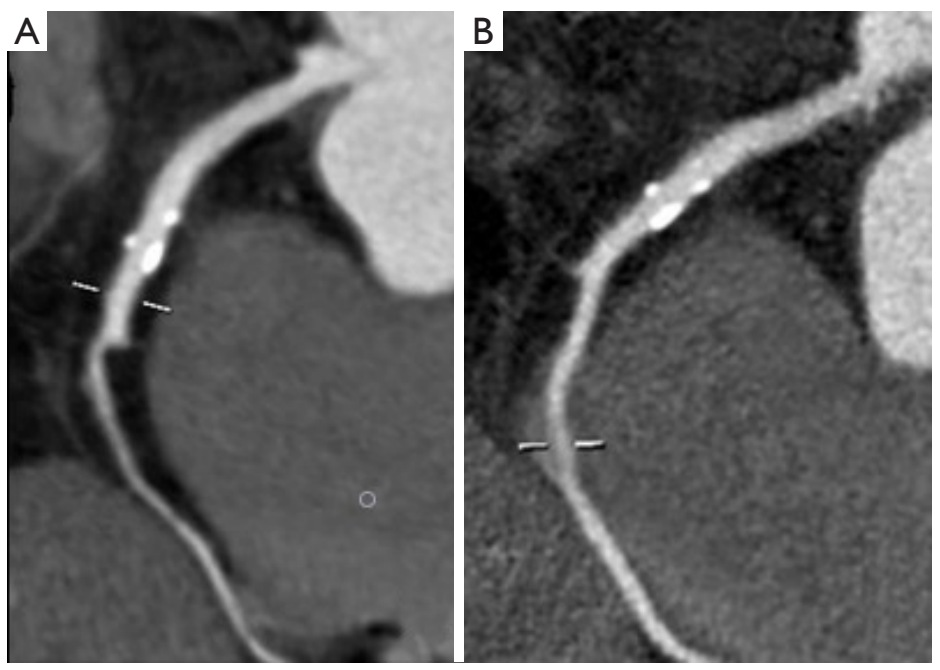

Figure 3 Multiplanar reconstructions of the right coronary artery displaying calcified plaques with smooth (A) and sharp filtering (B).

however larger matrix $(1,024 \times 1,024,2,048 \times 2,048)$ are now available, despite greater time required for reconstruction, transfer, process, display, and storage are needed (15).

Also filtering kernels and iterative reconstructions can both affect spatial resolution. Convolution filters are applied to reduce the blurring that occurs with back projection alone. Each convolution kernel uses the value of nearby pixels to create a filtered profile. There are different types of kernel filters that can be roughly classified in: standard, smooth and sharp. The type of filters determines spatial resolution and noise; noise increases with sharp kernels, instead spatial resolution improves with sharper kernels and worsens with smooth ones (Figure 3).

An increased spatial resolution is required for in-stent evaluation and for coronary calcifications. Kernels are used in the Filtered Back Projection (FBP); however, low dose protocols especially in obese patients cause an increased image noise (24).

Moreover, also the use of thinner slice reconstructions may improve spatial resolution, at the expenses of an increased noise given the remaining parameters constant $(25,26)$.

The iterative reconstructions (IR) have been developed to improve image quality and reduce noise $(27,28)$. IR starting from the images obtained from the FBP, generate new projection data that are compared to the original ones, then noise corrections are made. This process is repeated (i.e., iterated) several times (24). IR have the enormous advantage of reducing dose preserving a good image quality, as demonstrated in the literature (29). Yang et al. (30) demonstrated that IR improve in-stent restenosis evaluation in high pitch dual source CT (DSCT), with a better specificity $(82 \% v s .62 \%)$ and positive predictive value $(66 \%$ vs. $50 \%$ ) compared to FBP.

Concerning the use of IR for coronary artery calcium quantification, Schindler et al. (31) demonstrated that Agatston score quantification is not different between FBP and IR, even if risk reclassification may occur in a small subset of subjects. Instead, other studies showed a reduction in Agatston, volume and, to a lesser extent, mass scores quantified with IR versus FBP algorithm (31,32).

Concerning obese patients, obtaining good CCT image quality in this cohort can be a challenge, and in general requires more radiation dose to compensate for the increased noise. Wang et al. (33) demonstrated that IR compared to FBP (in routine protocol at $120 \mathrm{kV}$ ) allows a maintained image quality with significantly lower radiation dose $(4.41 \pm 0.83$ vs. $8.83 \pm 1.74 \mathrm{mSv})$ in obese patients.

As above discussed, IR have significant advantages, including radiation dose and noise reduction (Figure 4); however, IR have also some disadvantages. The reconstruction processes require longer times, which however have decreased in recent years; moreover, images obtained with IR have a different aspect and may cause some diagnostic misinterpretation in operators with insufficient experience (28).

The pitch is another element to be considered in spatial resolution; it corresponds to the speed of motion of the CT table per rotation of the gantry, divided by the amplitude of the detectors (34). Cardiac imaging in spiral mode requires low pitch because higher pitch determines 

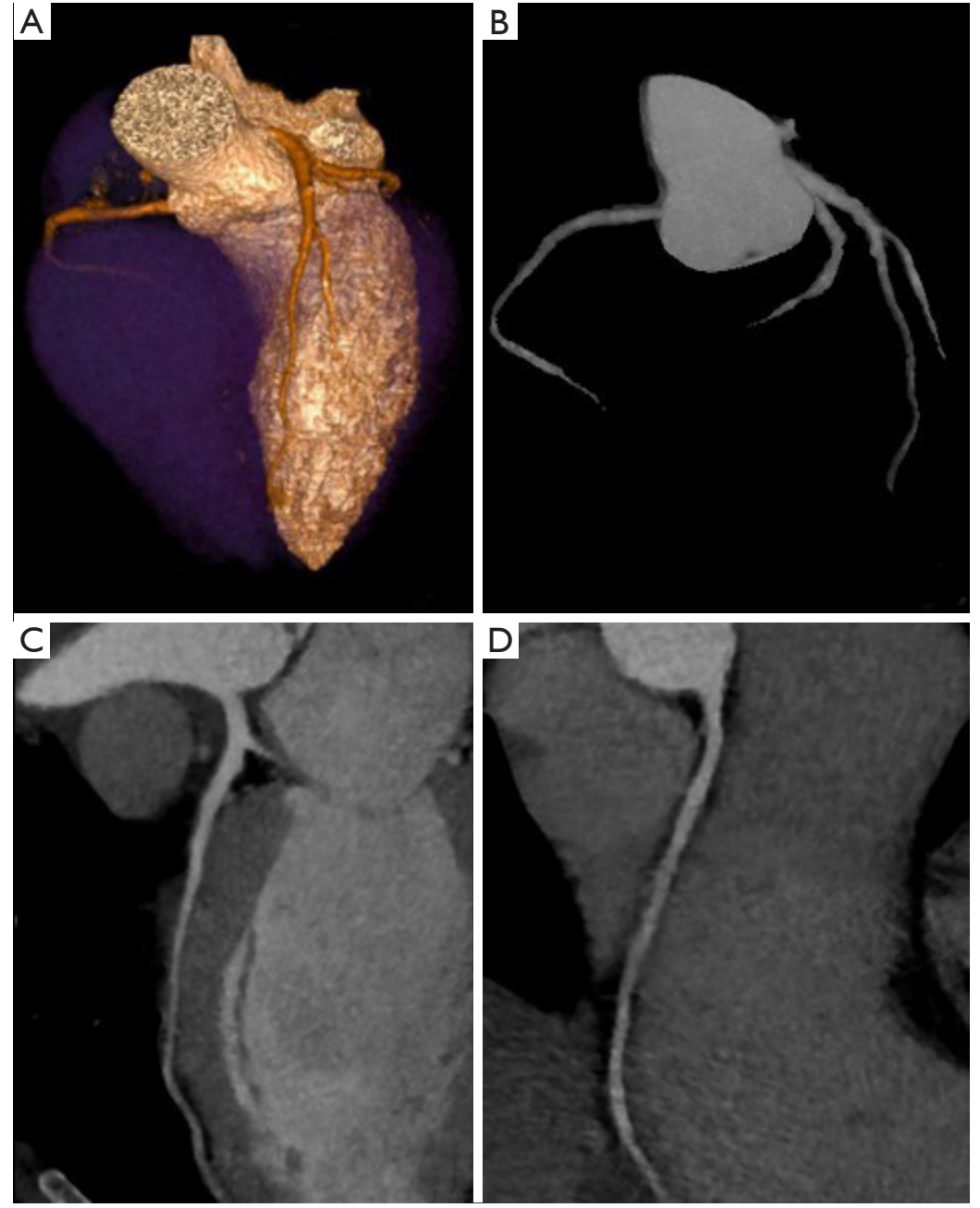

Figure 4 Cardiac CT with iterative reconstructions. (A) Volume rendering; (B) coronary artery tree map; (C,D) multiplanar reconstructions of the coronary arteries.

gaps, and high-quality 3D images with minimal artifacts require data overlap; a typical CCT pitch is between 0.2 and 0.4 depending on the heart rate of the patient during the scan (14). Furthermore, a low pitch helps increasing CT data redundancy along the cardiac cycle improving the possibility to find and use the best phase (i.e., the phase of the cardiac with less residual motion) for coronary artery assessment. However, it must always be considered that a low pitch requires an average increased radiation dose.

In summary, coronary stent evaluation poses problems related to their small size and the hyperdense material used; blooming artifacts determine false positives and lower specificity. The presence of high-density structures is responsible for hardening of the beam on the detector. Improving spatial resolution remains the best technique for reducing artifacts related to stent evaluation; the use of thin acquisition layers and small FOV are crucial. DECT, IR and sharp kernels are able to improve in-stent evaluation (35).

Yang et al. (30) demonstrated the value of IR in improving detection of in-stent restenosis by high-pitch DSCT, especially in stents of smaller dimensions. Geyer et al. (36), using a second generation DSCT upgraded with an integrated-circuit detector, studied the influence on coronary stent smaller than 3 millimetres together with IR; authors demonstrated that, in a heart-phantom movingmodel, IR improved sharpness, signal and contrast-to-noise ratio, reducing blurring and noise, if compared to FBP.

Main determinants of spatial resolution in CCT and potential improvements are summarized in Table 2. 
Table 2 Determinants of spatial resolution in CCT and potential improvements

\begin{tabular}{|c|c|c|}
\hline Factor & Issue & Solution \\
\hline Number of projection views & Improved sampling of detector units & Z-flying spot; tantalum grids in front of detectors \\
\hline Detector size & Reduced radiation dose efficiency & $\begin{array}{l}\text { Reduced detector size and septa area; photon-counting } \\
\text { detectors }\end{array}$ \\
\hline Matrix size & $\begin{array}{l}\text { Longer time for transfer, process, display, and } \\
\text { storage }\end{array}$ & Larger matrix sizes $(1,024 \times 1,024,2,048 \times 2,048)$ \\
\hline
\end{tabular}

CCT, cardiac computed tomography.

\section{Temporal resolution}

An adequate temporal resolution was another of the primary hardware issue to be solved for an adequate coronary imaging.

Temporal resolution is the time needed to acquire the data to generate an image (34).

Temporal resolution is less relevant for stationary organs, but it is essential for constantly moving organs evaluation, such as the heart. High temporal resolution is needed for CCT scan to reduce respiratory motion artifacts and to overcome cardiac motion artifacts.

The high temporal resolution is obtained mainly through a fast gantry rotation and, in some specific conditions, through a partial reconstruction of the scan. Temporal resolution is affected by the gantry rotation speed, image reconstruction (i.e., prospective $v s$. retrospective triggering), pitch and post-processing algorithm.

The gantry rotation time is the time required for the tube and the detectors to perform a full 360 degrees rotation. Gantry rotation time limits temporal resolution; the quicker the tube rotation, the higher the temporal resolution. For CCT we must bear in mind that it is sufficient to obtain half a rotation of the gantry to generate one image $\left(180^{\circ}\right.$ reconstruction algorithm). Since the gantry rotation time cannot be easily accelerated, other solutions have been developed to improve the temporal resolution obviating this technical limit (14).

In the last decades, the different CT scanner generations provided a progressive temporal resolution improvement. The first studies on cardiac imaging, for the evaluation of coronary calcifications, were carried out with electron beam computed tomography (EBCT). EBCT is a tomographic technique made by an electron beam projected against a tungsten ring positioned under the patient's table. The impact of the electrons determines the emission of an $\mathrm{X}$-ray fan that is rotated around the patient's body. This is a method with a very high temporal resolution $(50 \mathrm{~ms})$, but with a low spatial resolution $(3 \mathrm{~mm})(37)$. EBCT was abandoned due to the construction complexity and the high equipment costs. In this regard, the introduction of multi-slice CT has revolutionized coronary imaging, with considerable reduction also in the patient's breath-holding.

Multi-slice CT is based on the same volumetric scanning principle as spiral CT. With the increase in the number of slices that can be acquired simultaneously during a rotation of the X-ray tube, the acquisition speed of a volume increases. The concomitant increase in the rotation speed of the X-ray tube, introduced with multi-slice CT, allowed an increase in temporal resolution, bringing it to values in the order of $250 \mathrm{~ms}$ (for a gantry rotation time of $500 \mathrm{~ms}$ ); such temporal resolution allowed a reduction of diastolic motion of the heart.

Several protocols are available for CCT imaging, each protocol has several advantages and disadvantages related to the dose and reconstruction feasibility.

Research has concentrated on the realization of protocols able to reduce the dose as much as possible, while maintaining high diagnostic accuracy and manageability of reconstructions.

During prospective ECG triggered acquisition, the tube is activated only in a selected phase of the RR interval; this is a sequential (i.e., step-and-shoot) type of acquisition, with a temporal resolution from 200 to $250 \mathrm{~ms}$ (for a gantry rotation time of 400 to $500 \mathrm{~ms}$ ). Image datasets are limited, but it allows a considerable reduction of the dose compared to the retrospective protocol (14). In prospective ECG-triggered sequential CT a volume/stack of images is 
reconstructed starting from different images block acquired in axial scan by different heartbeats. In prospective ECGtriggered protocol each images stack is related to the number of active detector slices and it is affected by the detector z-coverage or width (14). In prospective ECGtriggered scan (i.e., step-and-shoot scan mode) the tube is activated only in a certain phase of the cardiac cycle with a minimum acquisition window; then, the table moves for further acquisition. This technique increases the nominal pitch to approximately 1 with a reduced overlap and a significant dose reduction (4); this is unless a larger diastolic acquisition window is required, in which case the flexibility of the reconstruction phase is much higher and also the radiation dose increases.

On the other side, with the retrospective ECG-gated protocols (i.e., spiral) the effective temporal resolution is still the same (between 200 and $250 \mathrm{~ms}$ for a gantry rotation time of 400 to $500 \mathrm{~ms}$ ); however, in special technical conditions, it is possible to split the reconstruction stacks in different neighbouring cardiac cycles and create a virtual reduction of temporal resolution of $80 \mathrm{~ms}$. During spiral CCT, $\mathrm{X}$-rays are active continuously throughout the scan range of the heart. The radiation dose delivered is higher as compared to the one needed in prospective ECGtriggered protocols, but it allows obtaining more redundant data, both on the systolic and diastolic phase (i.e., for cardiac function assessment) (14). Retrospective ECGgated spiral scan with ECG-controlled tube current (milliAmpere) modulation is a type of retrospective acquisition in which the tube power is maximal in a selected phase of the cardiac cycle (i.e., generally mid-to-end diastole) and it is considerably reduced during the other phases. The technique has the advantage of the retrospective acquisition, but with radiation dose reduction of $30-50 \%$ depending on the heart rate of the patient (4).

On the other hand, the image reconstruction algorithms depend on the availability of segments to reconstruct an image. The $180^{\circ}$ of rotation required to generate an image can be obtained from a single cardiac cycle (i.e., single segment reconstruction or partial scan acquisition) or from several consecutive cardiac cycles (i.e., multisegment reconstruction; see also above). Therefore, the reconstruction methods affect the temporal resolution both in prospective and retrospective ECG acquisitions. Since partial scan reconstruction can be used both for prospective and retrospective acquisition (effective resolution equivalent to half of the rotation time of the gantry), multi-segment reconstruction has been developed to overcome issues related to the poor gantry rotation time, but it is exploitable only with retrospective acquisition method. Multisegment reconstruction uses some portions of projections from different heartbeats; these data are then collected to compose a full reconstruction, reaching a higher temporal resolution (14).

One of the greatest innovations of the past 12 years in the field of CCT with massive impact on effective temporal resolution was the introduction of a second $\mathrm{X}$-ray tube (and a second detector) within the same CT scanner; this is called Dual Source CT (DSCT), and allowed an instant doubling of temporal resolution. A DSCT scanner contains two sets of tubes/detectors, arranged at approximately 90 degrees angles to each other. DSCT requires only a quarter turn of the gantry $\left(90^{\circ}\right.$ rotation; $\left.33 \mathrm{~ms}\right)$, if partial scan reconstruction is used. The increased temporal resolution of DSCT allows CCT imaging at much higher heart rates (38). The latest generations of DSCT achieve effective temporal resolution between $83 \mathrm{~ms}\left(2^{\text {nd }}\right.$ generation DSCT) and $66 \mathrm{~ms}\left(3^{\text {rd }}\right.$ generation DSCT) depending on the scanner generation $(19,24)$.

The $1^{\text {st }}$ generation of DSCT had some constraints due to the physical space within the gantry and the $2^{\text {nd }}$ detector, it could not be as large as the primary detector, determining a reduced FOV when used together (39). With the last generation of DSCT this issue has been addressed. DSCT has several additional advantages: due to the super-fast effective temporal resolution the pitch can be increased (instead of reduced as it happens with single source CT) at higher heart rates, decreasing dose (19) and allowing long anatomic coverage; ECG-synchronized scan is more flexible (40) and multisegment reconstruction is not necessary due the extremely high temporal resolution (21).

It is not clear whether we will be able to improve the temporal resolution to such an extent that we do not need an acquisition synchronized to the ECG trace. The best scenario would be to improve the temporal resolution to such an extent. The introduction of $2^{\text {nd }}$ and $3^{\text {rd }}$ generation DSCT allowed a high-pitch single-heartbeat acquisition with the scan of the whole heart volume in a single cardiac cycle. The main advantage of this protocol was the significant radiation dose reduction (i.e., $<1 \mathrm{mSv}$ ). The exposure was about $1 / 10$ of that of a conventional retrospectively gated spiral scan and about one third of that of a prospectively ECG-triggered scan (24). In that perspective, 320-row CT scanners allow also the acquisition of the whole heart in a single heartbeat. The entire $z$-axis of the heart is covered by a single detector array (i.e., $16 \mathrm{~cm}$ 
Table 3 Determinants of temporal resolution in CCT and potential improvements

\begin{tabular}{lcc}
\hline Factor & Issue & Solution \\
\hline Gantry rotation time & Mechanical limits; tube power/radiation dose & $\begin{array}{c}\text { Shortest gantry rotation time 50-100 ms (above } \\
25 \text { ms of coronary angiography) }\end{array}$ \\
Novel scanner concepts & Cost; tube power/radiation dose & Dual source computed tomography \\
New software solutions & Arrhythmias; tube power/radiation dose & $\begin{array}{c}\text { Multisegment reconstruction approaches } \\
\text { Iterative reconstruction algorithms }\end{array}$ \\
\hline
\end{tabular}

$\mathrm{CCT}$, cardiac computed tomography.

width) (24); however, given the standard effective temporal resolution the radiation dose is much higher.

Main determinants of temporal resolution in CCT and potential improvements are summarized in Table 3.

\section{Post processing analytics and advanced tools}

Among the future applications of CCT, there is a shift from stenosis assessment to more functional and radiomics features, such as atherosclerotic plaque characterization and quantification, hemodynamic assessment of stenosis, dynamic myocardial perfusion and epicardial adipose tissue (EAT) assessment. This new era in cardiac CT should be possible only with novel hardware and software solutions.

The radiation dose issue, however, remains one of the most important to investigate. The concept of radiation dose reduction to minimize the dose to as low as reasonably achievable (ALARA principle) is particularly complex in CCT, since the dose is strictly related to image quality and the capability of proper coronary visualization. Furthermore, the improvements in spatial and temporal resolution have led in the past to an increase in tube power and radiation dose to the patient. The issue is to obtain images of diagnostic quality, reducing as much as possible the radiation dose. The radiation dose is influenced by several factors even related to the patient itself: clinical indication, body mass index (BMI), heart rate and betablockers administration. Clinical indications influence the scan length or z-axis coverage; therefore, the scan length should be as narrow as possible, in order to reduce the radiation dose. Patients with a high BMI or with fat predominance in the upper portion of the body require higher values of $\mathrm{kV}$ and $\mathrm{mA}$ to obtain diagnostic images. BMI strictly influence acquisition parameters: tube current and tube voltage. There is a linear relationship between the dose delivered to the patient and the tube current $(\mathrm{mA})$. Tube voltage $(\mathrm{kVp})$ has indeed an exponential relationship to the radiation exposure (40). Tube current is controlled by automatic exposure control (AEC), which adapts the $\mathrm{mA}$ automatically in both angular and longitudinal direction according to the patient's size, allowing a dose reduction of $33 \%$, without hampering image quality (41). Therefore, AEC must never be deactivated. Another method for dose reduction in CCT is to decrease the tube voltage $(\mathrm{kVp})(40)$. The main disadvantage of $\mathrm{kV}$ reduction is the reduction of the diagnostic accuracy and noise increase; however, if the tube power is adequate this can be easily compensated by an increased $\mathrm{mA}$ delivery with still a consistent reduction of radiation dose.

Heart rate is also important because some radiation dose reduction protocols can be used only if the heart rate is low (i.e., prospective ECG-gated scan). Heart rate and available scanner technology determine the choice of the examination protocol.

The dose is also influenced by some factors strictly related to the CT scanner: type of scanner, acquisition technique and parameters, and operator experience. The introduction of several CT protocol developments and software modifications such as ECG-pulsing, automatic tube potential selection, tube current modulation and IR (24) have the aim to obtain diagnostic images lowering the dose. It has been shown that the use of ECG modulation of milliAmpere can reduce the dose up to $50 \%$ depending on the patient's heart rate (42).

In certain situation, in particular in non-obese patients with a low heart rate, prospectively ECG-triggered highpitch spiral coronary CCT may be performed below $1 \mathrm{mSv}$ maintaining an excellent image quality evaluated by image quality score (43). Also, the use of IR allows a dose reduction, if compared to FBP, especially through a tube current reduction, but maintaining a high image quality and diagnostic accuracy (44).

Contrast resolution is another of the primary issues in CCT together with temporal and spatial resolution. 
Contrast resolution is the ability to distinguish two different adjacent objects based on their attenuation values. Several factors influence contrast resolution: detector type, patient features, $\mathrm{mA}, \mathrm{kVp}$, slice thickness, convolution filters, IR, and contrast media administration protocols (34). These parameters also affect image noise, which determines the overall quality of the exam. The type and speed of administration of contrast agent is a main factor influencing image quality in CCT (45). The right antecubital vein is preferable because of anatomical issues; small volume of high-concentrated iodinated contrast agent at a high injection rate is recommended, because it results in an earlier arterial peak enhancement fundamental for the visualization of the coronary arteries. Reducing the tube voltage to $100 \mathrm{kVp}$ or even $70-80 \mathrm{kVp}$ in selected patients allows a reduction of contrast medium volume and better visualization of coronary vessels (45). Furthermore, improvement in anti-scatter grids could further improve the signal-to-noise ratio and overall image quality (46).

Evolutions in the tube construction, with a rapid $\mathrm{kVp}$ switching mechanism, could further reduce the noise, especially in DECT (47). The implementation of IR allows a reduction of $50 \%$ to a pooled effective dose with preserved quality of imaging (48). The introduction of spectral CT through DSCT has made it possible to better differentiate the contrast medium from other hyperdense materials. Among the potential upgrades of DSCT, better X-ray filtration could allow better spectral separation of materials. Some steps forward have been made with new types of CT contrast compounds. First, some contrast media were designed to preferentially accumulate on atherosclerotic plaques macrophages $(49,50)$ or during HDL-macrophage pathway with gold nanoparticles (51); however, these experiments have several limitations on the human model for potential cytotoxicity $(50,51)$. Second, spectral CT would benefit from new biocompatible high-atomic number contrast materials with different biodistribution such as tungsten, tantalum, or bismuth $(52,53)$.

Within the new applications of CCT we can include the identification of intracardiac thrombi.

Thrombus assessment may be required before an interventional procedure or in the search for an embolic source in cryptogenic stroke (54).

In clinical practice transesophageal echocardiography (TEE) is recommended for this purpose (55) but in the last years CCT demonstrated its ability to identify intracavitary thrombi.
In a meta-analysis, Romero et al. (56) analyzed the diagnostic accuracy of CCT, in comparison to TEE, for left atrial or appendage thrombi identification; mean sensitivity and specificity were respectively of $96 \%$ and $92 \%$, with a positive and negative predictive value of $41 \%$ and $99 \%$, instead diagnostic accuracy was $94 \%$.

The use of CCT as an alternative to trans-esophageal echocardiography eliminates some risks such as bleeding and esophageal perforation.

CCT, with retrospective protocols, allows also the evaluation of left ventricular volumes and ejection fraction without additional radiation dose (Figure 5).

Several software are commercially available for cardiac function evaluation; the optimal left ventricle opacification during the angiographic phase allows an excellent definition of cardiac contours for the precise differentiation of left ventricular wall from blood, so allowing precise volumes evaluation (57).

A high spatial resolution is not necessary for the evaluation of ventricular function, while high temporal resolution is very important.

The software automatically identifies the end-systolic and end-diastolic phase, calculating the volume between the mitral valve plane and the cardiac apex.

Software is generally based on predefined threshold levels. If contrast medium density in the ventricular lumen is not adequate, endocardial contours must be drawn manually (34).

The CONFIRM study evaluated the role of left ventricle ejection fraction and volume assessment in risk evaluation for incident mortality; the study demonstrated that a worsen left ventricular ejection fraction, calculated by CCT, was associated with mortality so ameliorating risk classification and confirming the role of CCT in calculating cardiac function (58).

Within novel applications of CCT, there is a prominent role in transcatheter aortic valve implantation (TAVI) preoperative assessment (59) (Figure 6); CCT has the enormous advantage of allowing simultaneous evaluation of coronary arteries and their ostia height, aortic valve anatomy, vascular calcifications together with main vascular access.

\section{Future perspectives}

Some novel software applications have been implemented in CCT in the last years thanks to technological improvements.

The future of cardiac imaging will integrate already 

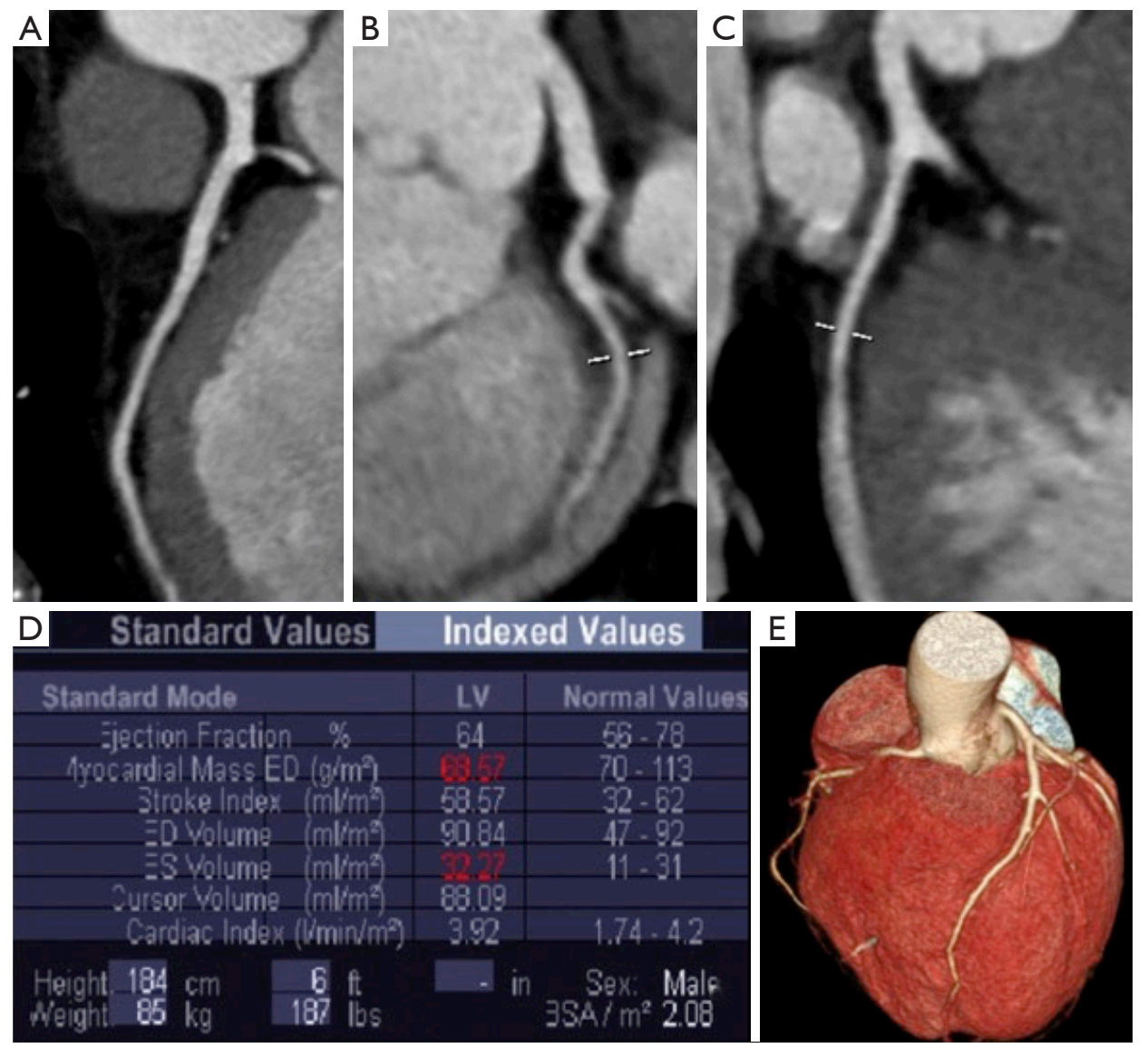

Figure 5 CT of a patient with normal coronary arteries who underwent cardiac computed tomography (CCT) with retrospective ECGgated helical scan with ECG-controlled tube current modulation: multiplanar reconstructions of coronary arteries (A,B,C); indexed left ventricular function (D) and volume rendering (E).

existing measures with new tools (60), but the future passes through technological progress.

In particular, fractional flow reserve (FFR) coupled with CCT has recently emerged as a great innovation to define hemodynamic significance of stenoses and potential candidates to revascularization $(61,62)$, with standard protocols and without additional radiation dose or medication (Figures 7-9).

In this setting, the PLATFORM study showed that the use of FFR-CT was associated with a significantly lower rate of ICA procedures showing non-obstructive coronary artery disease (63). Moreover, the SYNTAX III Revolution trial showed an additional advantage of CCT in the planning of complex revascularization (64). Another relatively novel method to assess potentially significant lesions is represented by CT perfusion (CTP) (Figure 10). CTP applications derive from the improved spatial and temporal resolution of recent scanners. The basic principle is the visualization of myocardium areas with reduced or delayed contrast enhancement, even under stress conditions. In this setting, DECT acquisition allows iodine map analysis of myocardial perfusion with measures of per-voxel iodine concentration (65). FFR-CT and CTP demonstrated similar results in detecting flow-limiting stenoses, using ICA plus invasive FFR as the reference standard (66).

The technological advances of the last decade, together with the improvement of spatial and temporal resolution allowed the analysis of epicardial adipose tissue (EAT) characteristics (Figure 11).

Several semiautomatic software have been developed for the extraction and analysis of epicardial fat: completely manual approached and semi-automatic ones.

EAT extraction is based on the evaluation of density intervals; some of the most used methods are atlas-based ones (67) and machine-learning; the latter is achieving 

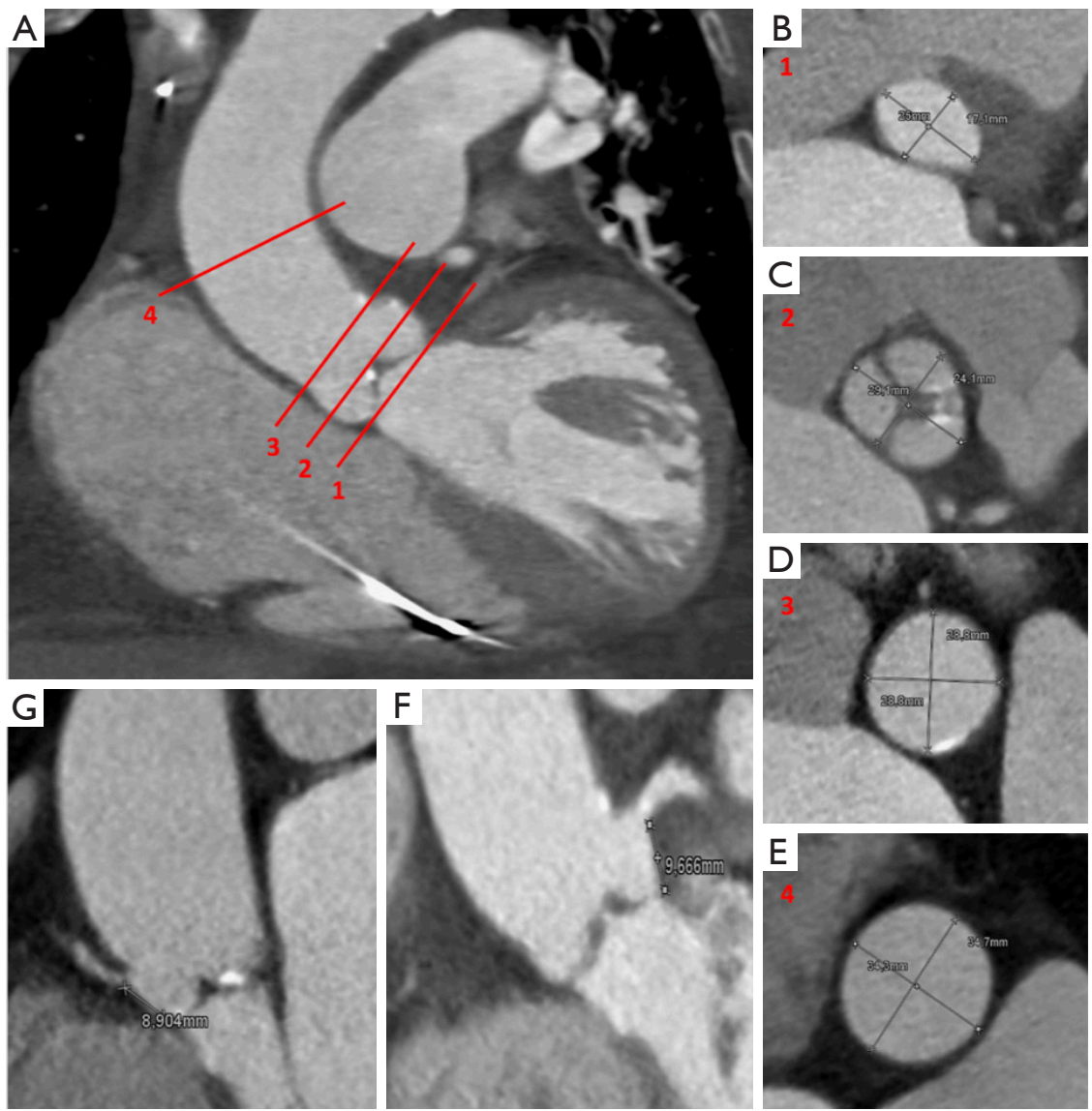

Figure 6 Role of computed tomography in evaluation of patients potentially candidates for transcatheter aortic valve implantation (TAVI): multiplanar reconstruction of ascending aorta with its anatomic landmarks (A-E): virtual basal ring (line 1 in panel A and panel B), valve plane (line 2 in panel A and panel C), sino-tubular junction (line 3 in panel A and panel D), ascending aorta (line 4 in panel A and panel E). Panel F represents the height of left coronary ostium, panel G represents the height of right coronary ostium.

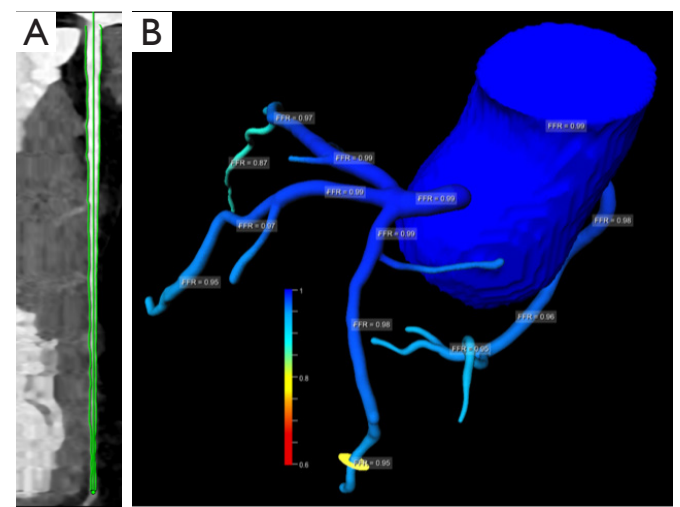

Figure 7 Example of FFRCT in a normal left anterior descending artery (LAD). A 55-year-old man with hypertension and atypical chest pain. (A) Normal LAD; (B) FFRCT three-dimensional model with different coronary segments FFRCT values (Software FFRct prototype by SIEMENS Frontier). excellent results.

Some of the proposed methods in the literature allow the evaluation of epicardial fat volume, density (also divided into quartiles) and thickness (Figure 12) (68).

However, the extraction of epicardial fat requires attention in choosing the extraction density interval, which varies between Calcium Score and angiographic phase (69).

Machine learning (ML) is one of the research fields of recent years; $M L$ includes computer algorithms that learn rules from large datasets, with no previous assumptions; machine learning has been used in cardiac imaging mainly to predict prognostic or diagnostic outcomes, but also for the analysis of extrapolated images for outcome prediction (70).

Motwani et al. studied the accuracy of machine learning to predict 5-year all-cause mortality in patients included in the CONFIRM registry who underwent CCT. Authors 

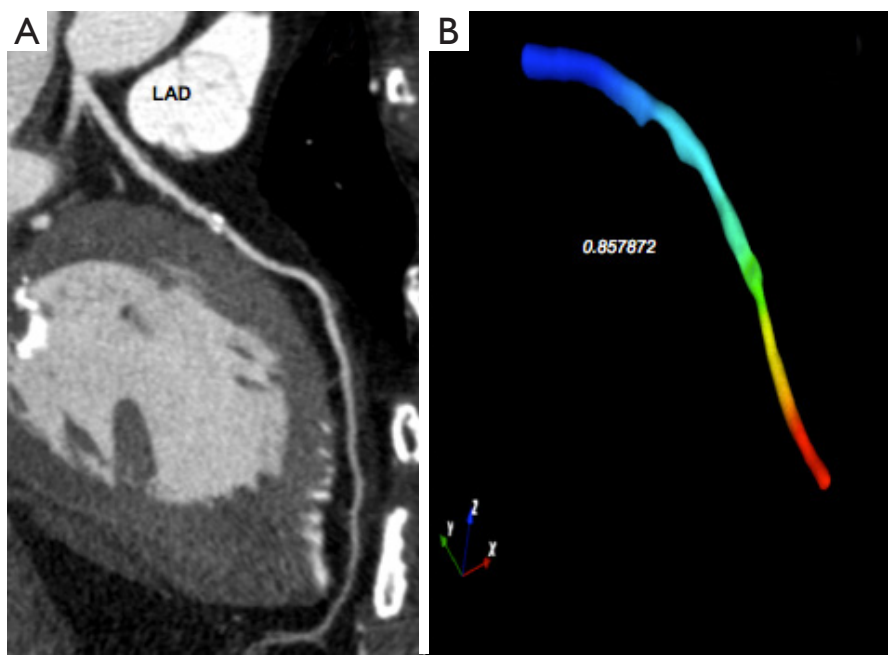

Figure 8 FFRCT in a diseased left anterior descending artery (LAD). A 73-year-old female with family history of cardiac heart disease, diabetes (insuline), dislipidemia, hypertension (under treatment) and typical effort angina. (A) A coronary calcified plaque in LAD, (B) an FFRCT value of 0.857872 (SmartFFR, SMARTool platform for clinical decision support system-CDSS).
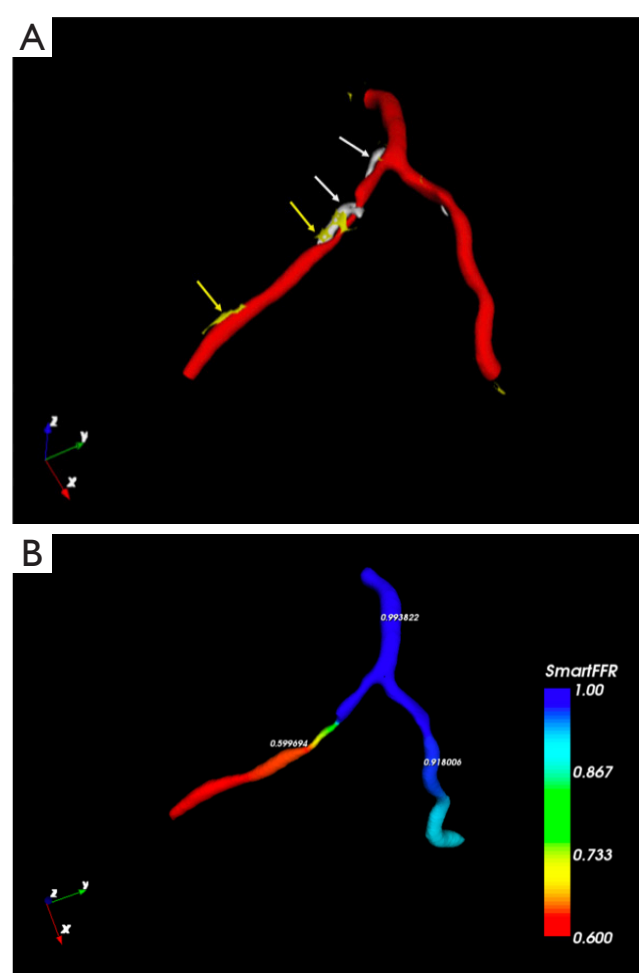

Figure 9 Cardiac computed tomography (CCT) 3D reconstruction of the left coronary artery with plaque components: white arrows indicate calcified elements of the plaque, instead yellow arrows indicate non-calcified ones (A) and fractional flow reserve (FFR) values (B) (SmartFFR, SMARTool platform for CDSS) showing a left anterior descending coronary flow-limiting stenosis with a value of 0.599694 . demonstrated in a population of 10,030 patients that $\mathrm{ML}$ was superior to CCT or clinical data alone in predicting mortality (71).

Machine learning may allow identifying individual risk factors on which a targeted therapy can be carried out.

A sub-study of the NXT trial analysed 254 patients, who underwent CCT and ICA, demonstrating that an objective combination of clinical data and quantitative CCT plaque measures obtained by machine learning ameliorated the prediction of hemodynamic significance of coronary lesions measured by invasive fractional flow reserve. ML exhibited also a higher area-under-the-curve (0.84) than individual CCT measures alone (72).

Sakellarios et al. used multi-disciplinary human data for the development of predictive models based on machine learning and computational biomechanics demonstrating the efficiency of the platform (73).

The SMARTool (Simulation Modeling of coronary ARTery disease) was designed to predict risk for primary and secondary prevention of coronary disease. It aims to develop a cloud-based platform for the clinical decision support (CDSS) of patients with CAD and it has been obtained integrating clinical, molecular, biohumoral and CCT imaging data from 263 patients into predictive risk models of CAD progression (74).

Deep learning is a part of the family of ML and can be used for the amelioration of image quality (75).

Tatsugami et al. (76) compared the image quality of CCT 

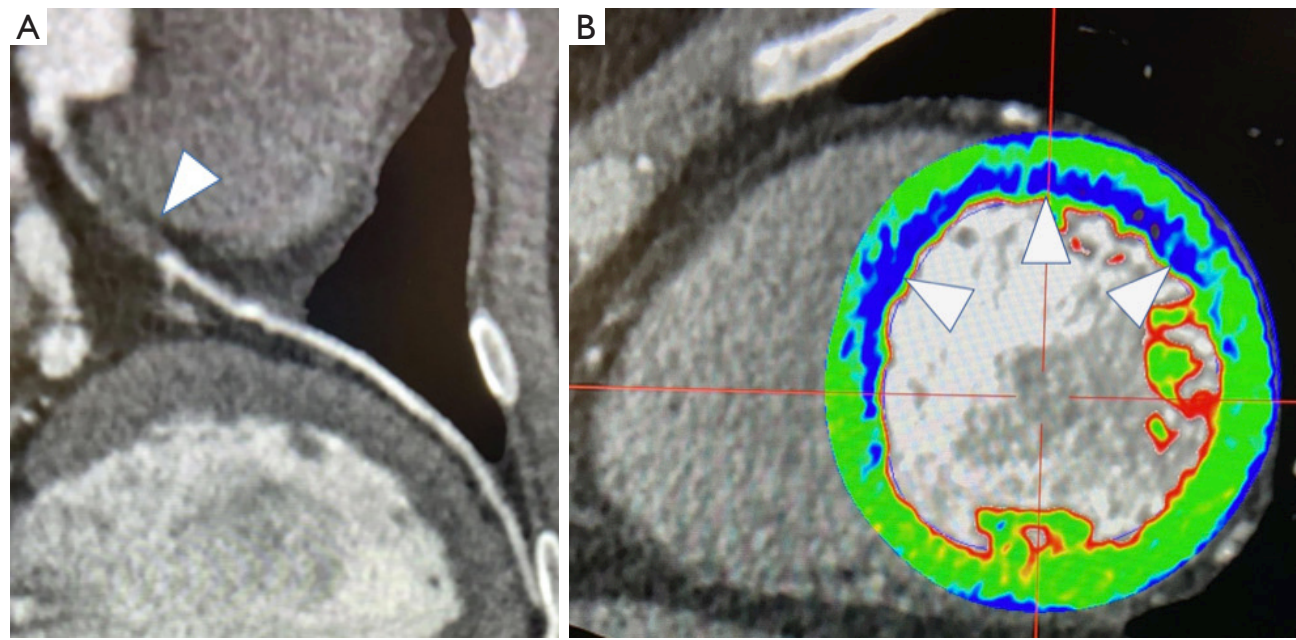

Figure 10 Example of CT perfusion in a 36-year-old man with proximal left anterior descending artery (LAD) non calcified plaque determining segmental (sub)occlusion as indicated by the arrowhead (A) and subendocardial perfusion defect, as indicated by the arrowhead (B) during rest CCT acquisition.
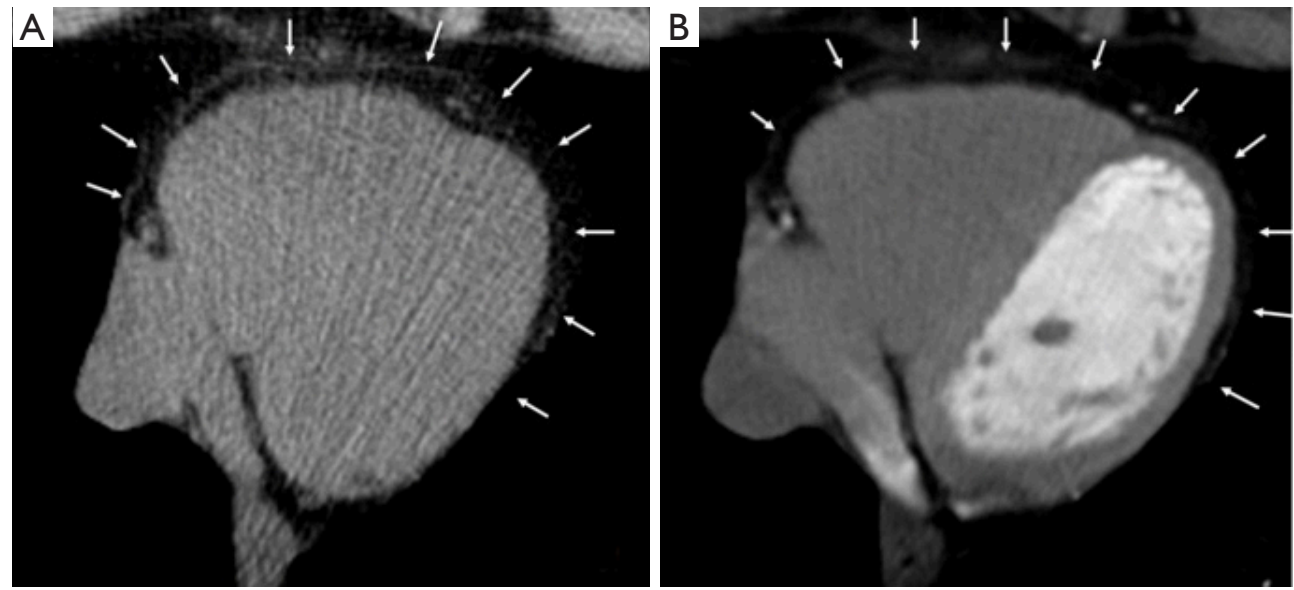

Figure 11 Epicardial adipose tissue surrounded by the pericardium (arrows) in cardiac computed tomography (CCT): in Calcium Score (A) and angiographic phase (B).

datasets subjected to deep learning-based image restoration (DLR) method with images subjected to hybrid iterative reconstruction and founded that DLR datasets had lower noise with a higher signal-to-noise ratio so showing that DLR are able to ameliorate image quality in CCT (76).

Lastly, the emerging field of radiomics software needs a brief mention. Radiomics aim to assess spatial, geometrical and tissue properties which cannot be identified by the human eye. Such applications are supposed to address in the future the characteristics of unstable coronary plaques (77).

\section{Conclusions}

In this review, we focused on the current approaches to improve spatial and temporal resolution of CT scanners and related technical and practical limitations. CCT achieved great results in anatomic imaging, even with a substantial reduction of the radiation dose. Nonetheless, hardware and software solutions should be implemented to address the functional significance of coronary artery stenosis and their need of revascularization. 

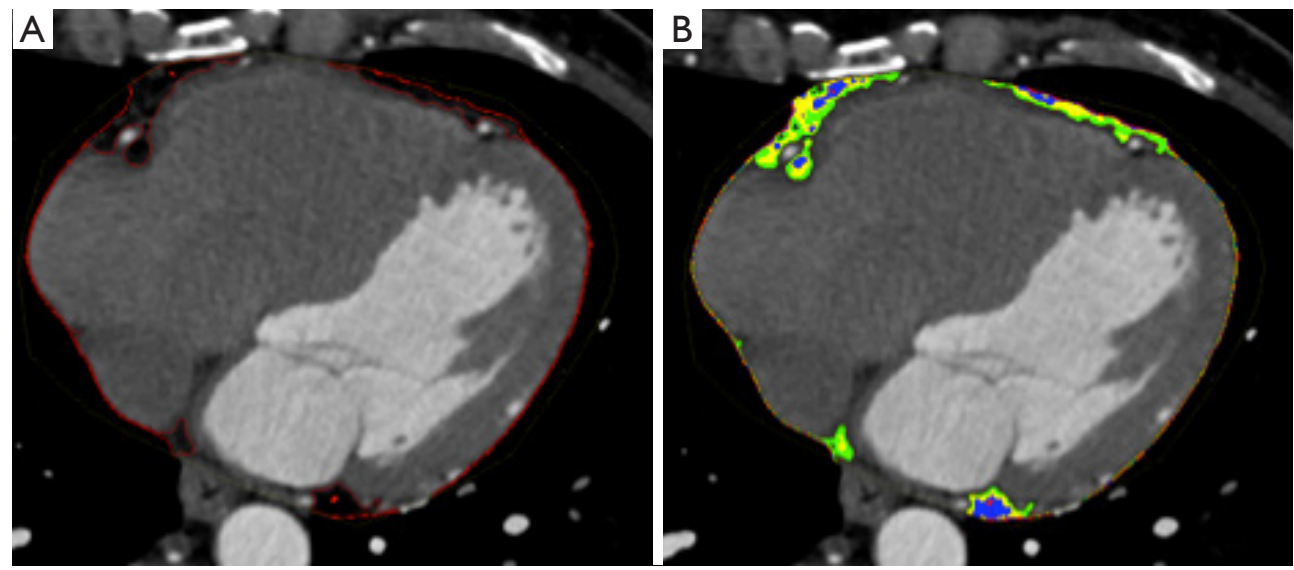

Figure 12 Semi-automatic software for epicardial fat evaluation: epicardial fat delineation (A) and volume evaluation (B) in cardiac computed tomography (CCT) datasets.

\section{Acknowledgments}

Funding: None.

\section{Footnote}

Provenance and Peer Review: This article was commissioned by the editorial office, Cardiovascular Diagnosis and Therapy for the series "Impact of Cardiac CT in Clinical Practice". The article has undergone external peer review.

Reporting Checklist: The authors have completed the Narrative Review reporting checklist. Available at http:// dx.doi.org/10.21037/cdt-20-527

Conflicts of Interest: All authors have completed the ICMJE uniform disclosure form (available at http://dx.doi. org/10.21037/cdt-20-527). The series "Impact of Cardiac CT in Clinical Practice" was commissioned by the editorial office without any funding or sponsorship. FC served as the unpaid Guest Editor of the series and serves as an unpaid editorial board member of Cardiovascular Diagnosis and Therapy from Jul 2019 to Jun 2021. The authors have no other conflicts of interest to declare.

Ethical Statement: The authors are accountable for all aspects of the work in ensuring that questions related to the accuracy or integrity of any part of the work are appropriately investigated and resolved.

Open Access Statement: This is an Open Access article distributed in accordance with the Creative Commons Attribution-NonCommercial-NoDerivs 4.0 International License (CC BY-NC-ND 4.0), which permits the noncommercial replication and distribution of the article with the strict proviso that no changes or edits are made and the original work is properly cited (including links to both the formal publication through the relevant DOI and the license). See: https://creativecommons.org/licenses/by-nc-nd/4.0/.

\section{References}

1. Cademartiri F, Runza G, Belgrano M, et al. Introduction to coronary imaging with 64-slice computed tomography. Radiol Med 2005;110:16-41.

2. Fattori R. La TC multidetettore nella diagnostica cardiovascolare. Milano, Italia: Springer, 2006.

3. Machida H, Tanaka I, Fukui R, et al. Current and Novel Imaging Techniques in Coronary CT. RadioGraphics 2015;35:991-1010.

4. Goldman LW. Principles of CT: Multislice CT. J Nucl Med Technol 2008;36:57-68.

5. Wang J, Fleischmann D. Improving Spatial Resolution at CT: Development, Benefits, and Pitfalls. Radiology 2018;289:261-2.

6. Flohr TG, Raupach R, Bruder H, et al. Cardiac CT: How much can temporal resolution, spatial resolution, and volume coverage be improved? J Cardiovasc Comput Tomogr 2009;3:143-52.

7. Schuleri KH, George RT, Lardo AC. Applications of cardiac multidetector CT beyond coronary angiography. Nat Rev Cardiol 2009;6:699-710. 
8. Faggioni L, Paolicchi F, Neri E. Elementi di tomografia computerizzata. Milan, Italia: Springer, 2011.

9. Available online: Healthcare-in-europe.com

10. Halliburton S, Arbab-Zadeh A, Dey D, et al. State-of-theart in CT hardware and scan modes for cardiovascular CT. J Cardiovasc Comput Tomogr 2012;6:154-63.

11. Ohnesorge BM, Flohr TG, Becker CR, et al. Multi-slice and Dual-source CT in Cardiac Imaging Principles - Protocols Indications - Outlook. Berlino: Springer, 2006.

12. Goldman LW. Principles of CT and the evolution of CT technology. In: Goldman LW, Fowlkes JB, editors. Categorical course in diagnostic radiology physics: CT and US cross sectional imaging. RSNA, Oak Brook, 2000; 124.

13. Shefer E, Altman A, Behling R, et al. State of the Art of CT Detectors and Sources: A Literature Review. Curr Radiol Rep 2013;1:76-91.

14. Mahesh M, Cody DD. Physics of Cardiac Imaging with Multiple-Row Detector CT. RadioGraphics 2007;27:1495-509.

15. Onishi H, Hori M, Ota T, et al. Phantom study of instent restenosis at high-spatial- resolution CT. Radiology 2018;289:255-60.

16. Flohr TG, Stierstorfer K, Ulzheimer S, et al. Image reconstruction and image quality evaluation for a 64-slice CT scanner with z-flying focal spot. Med Phys 2005;32:2536-47.

17. Flohr TG, Stierstorfer K, Süss C, et al. Novel ultrahigh resolution data acquisition and image reconstruction for multi-detector row CT. Med Phys 2007;34:1712-23.

18. Tang X, Narayanan S, Hsieh J, et al. Enhancement of in-plane spatial resolution in volumetric Computed Tomography with focal spot wobbling - overcoming the constraint on number of projection views per gantry rotation. J Xray Sci Technol 2010;18:251-65.

19. Lin E, Alessio A. What are the basic concepts of temporal, contrast, and spatial resolution in cardiac CT? J Cardiovasc Comput Tomogr 2009;3:403-8.

20. McCollough CH, Leng S, Sunnegardh J, et al. Spatial resolution improvement and dose reduction potential for inner ear CT imaging using a $\mathrm{z}$-axis deconvolution technique. Med Phys 2013;40:061904.

21. Bushberg JT, Boone JM, Leidholdt EM, et al. The Essential Physics of Medical Imaging. Philadelphia: Lippincott Williams and Wilkins, 2011.

22. Chaikriangkrai K, Choi SY, Nabi F, et al. Important advances in technology and unique applications to cardiovascular computed tomography. Methodist Debakey Cardiovasc J 2014;10:152-8.
23. Leng S, Bruesewitz M, Tao S, et al. Photon-counting Detector CT: System Design and Clinical Applications of an Emerging Technology. Radiographics 2019;39:729-743.

24. Schoepf UJ. CT of the Heart. Human Press, 2019.

25. Kalisz K, Buethe J, Saboo SS, et al. Artifacts at Cardiac CT: Physics and Solutions. Radiographics 2016;36:2064-83.

26. Kumamaru KK, Hoppel BE, Mather RT, et al. CT angiography: current technology and clinical use. Radiol Clin North Am 2010;48:213-35, vii.

27. Willemink MJ, De Jong PA, Leiner T, et al. Iterative reconstruction techniques for computed tomography part 1: technical principles. Eur Radiol 2013;23:1623-31.

28. Halliburton SS, Tanabe Y, Partovi S, et al. The role of advanced reconstruction algorithms in cardiac CT. Cardiovasc Diagn Ther 2017;7:527-38.

29. Jiang H. Computed Tomography: Principles, Design, Artifacts, and Recent Advances. Bellingham: Society of Photo Optical, 2015.

30. Yang J, Yang X, De Cecco CN, et al. Iterative reconstruction improves detection of in-stent restenosis by high-pitch dual-source coronary CT angiography. Sci Rep 2017;7:6956.

31. Schindler A, Vliegenthart R, Schoepf UJ, et al. Iterative image reconstruction techniques for CT coronary artery calcium quantification: comparison with traditional filtered back projection in vitro and in vivo. Radiology 2014;270:387-93.

32. Willemink MJ, Takx RA, De Jong PA, et al. The impact of $\mathrm{CT}$ radiation dose reduction and iterative reconstruction algorithms from four different vendors on coronary calcium scoring. Eur Radiol 2014;24:2201-12.

33. Wang R, Schoepf UJ, Wu R, et al. Image quality and radiation dose of low dose coronary $\mathrm{CT}$ angiography in obese patients: sinogram affirmed iterative reconstruction versus filtered back projection. Eur J Radiol 2012;81:3141-5.

34. Cademartiri F, Casolo G, Midiri M. La TC del cuore nella pratica clinica. Italia: Springer-Verlag, 2007.

35. Mahnken AH. CT Imaging of Coronary Stents: Past, Present, and Future. ISRN Cardiol 2012;2012:139823.

36. Geyer LL, Glenn GR, De Cecco CN, et al. CT evaluation of small-Diameter coronary artery stents: Effect of an Integrated Circuit Detector with Iterative Reconstruction. Radiology 2015;276:706-14.

37. Cademartiri F, Runza G, La Grutta L, et al. Non-invasive evaluation of coronary calcium. Radiol Med 2005;110:506-22.

38. Petersilka M, Bruder H, Krauss B, et al. Technical principles of dual source CT. Eur J Radiol 2008;68:362-8. 
39. Flohr TG, Bruder H, Stierstorfer K, et al. Image reconstruction and image quality evaluation for a dual source CT scanner. Med Phys 2008;35:5882-97.

40. Gerber TC, Kantor B, McCollough CH. Radiation dose and safety in cardiac computed tomography. Cardiol Clin 2009;27:665-77.

41. Maffei E, Martini C, De Crescenzo S, et al. Low dose CT of the heart: A quantum leap into a new era of cardiovascular imaging. Radiol Med 2010;115:1179-207.

42. Abada HT, Larchez C, Daoud B, et al. MDCT of the coronary Arteries: feasibility of low-dose CT with ECGpulsed tube current modulation to reduce radiation dose. AJR Am J Roentgenol 2006;186:S387-90.

43. Achenbach S, Marwan M, Ropers D, et al. Coronary computed tomography angiography with a consistent dose below $1 \mathrm{mSv}$ using prospectively electrocardiogramtriggered high-pitch spiral acquisition. Eur Heart J 2010;31:340-6.

44. Leipsic J, Labounty TM, Heilbron B, et al. Estimated radiation dose reduction using adaptive statistical iterative reconstruction in coronary CT angiography: the ERASIR study. AJR Am J Roentgenol 2010;195:655-60.

45. Weininger M, Barraza JM, Kemper CA, et al. Cardiothoracic CT angiography: current contrast medium delivery strategies. AJR Am J Roentgenol 2011;196:W260-72.

46. Siewerdsen JH, Jaffray DA. Cone-beam computed tomography with a flat-panel imager: magnitude and effects of x-ray scatter. Med Phys 2001;28:220-31.

47. Yeh BM, FitzGerald PF, Edic PM, et al. Opportunities for new CT contrast agents to maximize the diagnostic potential of emerging spectral CT technologies. Adv Drug Deliv Rev 2017;113:201-22.

48. Den Harder AM, Willemink MJ, De Ruiter QM, et al. Dose reduction with iterative reconstruction for coronary CT angiography: a systematic review and meta-analysis. Br J Radiol 2016;89:20150068.

49. Hyafil F, Cornily JC, Rudd JH, et al. Quantification of inflammation within rabbit atherosclerotic plaques using the macrophage-specific CT contrast agent N1177: a comparison with 18F-FDG PET/CT and histology. J Nucl Med 2009;50:959-65.

50. Young VEL, Degnan AJ, Gillard JH. Advances in contrast media for vascular imaging of atherosclerosis. Imaging Med 2011;3:353-66.

51. Cormode DP, Jarzyna PA, Mulder WJ. Modified natural nanoparticles as contrast agents for medical imaging. Adv Drug Deliv Rev 2010;62:329-38.

52. Mongan J, Rathnayake S, Fu Y, et al. In Vivo
Differentiation of Complementary Contrast Media at Dual-Energy CT. Radiology 2012;265:267-72.

53. Rathnayake S, Mongan J, Torres AS, et al. In vivo comparison of tantalum, tungsten, and bismuth enteric contrast agents to complement intravenous iodine for double-contrast dual-energy CT of the bowel. Contrast Media Mol Imaging 2016;11:254-61.

54. Pathan F, Hecht H, Narula J, et al. Roles of Transesophageal Echocardiography and Cardiac Computed Tomography for Evaluation of Left Atrial Thrombus and Associated Pathology: A Review and Critical Analysis. JACC Cardiovasc Imaging 2018;11:616-27.

55. January CT, Wann LS, Alpert JS, et al. 2014 AHA/ACC/ HRS guideline for the management of patients with atrial fibrillation: a report of the American College of Cardiology/American Heart Association Task Force on Practice Guidelines and the Heart Rhythm Society. J Am Coll Cardiol 2014;64:e1-76.

56. Romero J, Husain SA, Kelesidis I, et al. Detection of left atrial appendage thrombus by cardiac computed tomography in patients with atrial fibrillation: a metaanalysis. Circ Cardiovasc Imaging 2013;6:185-94.

57. Singh RM, Singh BM, Mehta JL. Role of cardiac CTA in estimating left ventricular volumes and ejection fraction. World J Radiol 2014;6:669-76.

58. Arsanjani R, Berman DS, Gransar H, et al. Left ventricular function and volume with coronary CT angiography improves risk stratification and identification of patients at risk for incident mortality: results from 7758 patients in the prospective multinational CONFIRM observational cohort study. Radiology 2014;273:70-7.

59. Francone M, Budde RPJ, Bremerich J, et al. CT and MR imaging prior to transcatheter aortic valve implantation: standardisation of scanning protocols, measurements and reporting-a consensus document by the European Society of Cardiovascular Radiology (ESCR). Eur Radiol 2020;30:2627-50.

60. Nicol ED, Norgaard BL, Blanke P, et al. The Future of Cardiovascular Computed Tomography: Advanced Analytics and Clinical Insights. JACC Cardiovasc Imaging 2019;12:1058-72.

61. Taylor CA, Fonte TA, Min JK. Computational fluid dynamics applied to cardiac computed tomography for noninvasive quantification of fractional flow reserve: scientific basis. J Am Coll Cardiol 2013;61:2233-41.

62. Nørgaard BL, Leipsic J, Gaur S, et al. Diagnostic performance of noninvasive fractional flow reserve derived from coronary computed tomography angiography in 
suspected coronary artery disease: the NX'T trial (Analysis of Coronary Blood Flow Using CT Angiography: Next Steps). J Am Coll Cardiol 2014;63:1145-55.

63. Douglas PS, Pontone G, Hlatky MA, et al. Clinical outcomes of fractional flow reserve by computed tomographic angiography-guided diagnostic strategies vs. usual care in patients with suspected coronary artery disease: the prospective longitudinal trial of FFR(CT): outcome and resource impacts study. Eur Heart J 2015;36:3359-67.

64. Andreini D, Modolo R, Katagiri Y, et al. Impact of Fractional Flow Reserve Derived From Coronary Computed Tomography Angiography on Heart Team Treatment Decision-Making in Patients With Multivessel Coronary Artery Disease: Insights From the SYNTAX III REVOLUTION Trial. Circ Cardiovasc Interv 2019;12:e007607.

65. Seitun S, De Lorenzi C, Cademartiri F, et al. CT Myocardial Perfusion Imaging: A New Frontier in Cardiac Imaging. Biomed Res Int 2018;2018:7295460.

66. Pontone G, Baggiano A, Andreini D, et al. Stress Computed Tomography Perfusion Versus Fractional Flow Reserve CT Derived in Suspected Coronary Artery Disease: The PERFECTION Study. JACC Cardiovasc Imaging 2019;12:1487-97.

67. Rohlfing T, Brandt R, Menzel R, et al. Quo vadis, atlasbased segmentation? In: Handbook of Biomedical Image Analysis, 2005;435-486.

68. Militello C, Rundo L, Toia P, et al. A semi-automatic approach for epicardial adipose tissue segmentation and quantification on cardiac CT scans. Comput Biol Med 2019;114:103424.

69. Bucher AM, Joseph Schoepf U, Krazinski AW, et al. Influence of technical parameters on epicardial fat volume quantification at cardiac CT. Eur J Radiol 2015;84:1062-7.

70. Commandeur F, Goeller M, Dey D. Cardiac CT:

Technological Advances in Hardware, Software, and Machine Learning Applications. Curr Cardiovasc Imaging Rep 2018;11:19.

71. Motwani M, Dey D, Berman DS, et al. Machine learning for prediction of all-cause mortality in patients with suspected coronary artery disease: a 5-year multicentre prospective registry analysis. Eur Heart J 2017;38:500-7.

72. Dey D, Gaur S, Ovrehus KA, et al. Integrated prediction of lesion-specific ischaemia from quantitative coronary CT angiography using machine learning: a multicentre study. Eur Radiol 2018;28:2655-64.

73. Sakellarios A, Correia J, Kyriakidis S, et al. A cloud-based platform for the non-invasive management of coronary artery disease. Enterprise Information Systems 2020. doi: 10.1080/17517575.2020.1746975.

74. Sakellarios A, Siogkas P, Georga E, et al. A Clinical Decision Support Platform for the Risk Stratification, Diagnosis, and Prediction of Coronary Artery Disease Evolution. Annu Int Conf IEEE Eng Med Biol Soc 2018;2018:4556-9.

75. Nakamura Y, Higaki T, Tatsugami F, et al. Possibility of Deep Learning in Medical Imaging Focusing Improvement of Computed Tomography Image Quality. J Comput Assist Tomogr 2020;44:161-7.

76. Tatsugami F, Higaki T, Nakamura Y, et al. Deep learningbased image restoration algorithm for coronary CT angiography. Eur Radiol 2019;29:5322-9.

77. Kolossváry M, Kellermayer M, Merkely B, et al. Cardiac Computed Tomography Radiomics: A Comprehensive Review on Radiomic Techniques. J Thorac Imaging 2018;33:26-34.
Cite this article as: Toia P, La Grutta L, Sollami G, Clemente A, Gagliardo C, Galia M, Maffei E, Midiri M, Cademartiri F. Technical development in cardiac CT: current standards and future improvements-a narrative review. Cardiovasc Diagn Ther 2020;10(6):2018-2035. doi: 10.21037/cdt-20-527 